> Si les travaux des embryologistes du début du $\mathrm{XIX}$ siècle marquent la fin de la théorie de la préformation dans sa forme initiale, les premières tentatives expérimentales de la fin de ce même siècle conduisent à développer le concept d'œuf mosaïque - une vision préformationniste plus élaborée du développement - dans lequel l'embryon est constitué d'une mosaïque de territoires au devenir déterminé et soumis à un développement autonome. Cependant, en séparant des blastomères d'oursin aux stades 2/4 cellules, un jeune chercheur, Hans Driesch, montre que chacun des blastomères se développe pour former une larve complète et harmonieuse et que le jeune embryon est donc capable de régulation, invalidant ainsi le concept du développement mosaïque - qui prédisait le développement indépendant de deux hémi-embryons - et ouvrant de nouveaux champs d'investigation à l'embryologie moderne. <

\section{Hans Driesch et Ia fin de toute vision préformationniste du développement}

\section{Christophe Chanoine}

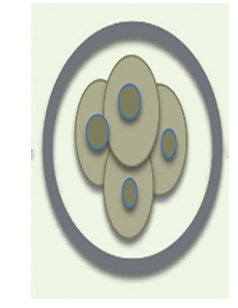

UMR Inserm 1124, université de Paris, Campus Saint-Germain-des-Près, faculté des sciences biomédicales et fondamentales, 45 rue des Saints-Pères, 75006 Paris, France.

christophe.chanoine@u-paris.fr

XVII ${ }^{e}$ siècle - en même temps que se développe les analyses utilisant la microscopie ${ }^{1}$ - par la théorie de la préformation qui s'imposa jusqu'au début du xixe siècle, sous des formes qui ont évoluées [2, 3].

À l'origine, les préformistes considèrent que l'être vivant provient d'un unique « germe », fourni par un seul parent, et qu'il est déjà complètement formé en miniature dans ce germe. En leur sein, apparaît rapidement un courant «spermiste» qui s'oppose à un courant «oviste». Pour les spermistes, seule la semence masculine joue un rôle, alors que pour les ovistes, le «germe» ne peut être qu'un œuf fourni par la mère. L'enthousiasme d'un savant comme Nicolas Hartsoeker (1656-1725) conduit l'imagination à prendre le pas sur l'observation microscopique : en 1694, il croit observer un petit homme - qu'il nomme homoncule - dans le spermatozoïde en position fœtale [4]. C'est cependant avec Jacques Gautier d'Agoty (1716-1785) que la théorie de la préformation atteint son apogée. Dans un petit pamphlet de 1750, intitulé Zôos-génésie, ou Génération de l'homme et des animaux, celui-ci explique avoir observé un homunculus dans les spermatozoïdes d'un homme, un tout petit cheval dans ceux d'un cheval, un tout petit âne (qui différait du cheval par la longueur des oreilles) dans ceux d'un âne, et un tout petit coq dans ceux d'un coq [5] !

Les préformistes restent longtemps majoritaires dans l'Europe des savants, avec des scientifiques de premier plan comme l'Allemand Gottfried Leibniz (16461716) et I'Italien Lazzaro Spallanzani (1729-1798).

Le premier microscope fut inventé en 1590 par les Hollandais Hans et Zacharias Janssen.
Dès l'Antiquité, les philosophes et les naturalistes se sont embryon (460-377 av. J-C), qui était médecin, et de son école, qui commencé à étudier le développement du pous travaux du philosophe Aristote (384-322 av. J-C). Dans son ouvrage, De la génération des animaux, écrit entre 330 et av. J-C, ce dernier émettait deux hypothèses, toutes les structures embryonnaires sont préformées et développement correspond à un simple accroissement de taille ; 2) au cours de l'ontogenèse, les nouvelles structures apparaissent progressivement, au cours d'un phénomène qu'il appelle épigenèse, c'est-à-dire «formation pardessus » [l]. C'est d'ailleurs cette dernière hypothèse qui prévaut jusqu'au Moyen-Âge, avant d'être supplantée, au

Vignette (๔ Laure Coulombel). 
Les voix s'opposant à la théorie de la préformation sont alors assez rares au XVIII siècle. C'est néanmoins le cas de Pierre Louis Moreau de Maupertuis (1698-1759) qui fait remarquer, en 1745, que l'enfant d'un noir et d'une blanche présente une couleur intermédiaire, et affirme finalement que le père et la mère ont une influence égale sur l'hérédité. Georges Louis Leclerc, Comte de Buffon (17071788), auteur d'une Histoire naturelle générale et particulière en 36 volumes publiés entre 1749 et 1767 , défend également des opinions épigénistes.

Mais ce sont trois noms qui restent associés au déclin de la théorie de la préformation dans sa forme initiale : le naturaliste allemand Caspar Wolff (1733-1794), ainsi que les deux embryologistes baltes, Heinz Christian Pander (1794-1865) et Karl Ernst von Baer (1792-1876).

La thèse intitulée Theoria Generationis, présentée en 1759 à l'Université de Halle par le jeune médecin allemand Caspar Friedrich Wolff (1734-1794), marque sans conteste un tournant dans les études embryologiques et dans l'opposition aux théories préformistes. Dans son remarquable ouvrage traitant du développement de l'intestin, De Formatione Intestinorum, publié en deux temps, en 1768 et en 1769, Wolf y présente une vision de l'épigenèse fondée sur des observations minutieuses des embryons de poulet, dans laquelle apparaît pour la première fois la notion de feuillet embryonnaire. Wolff montre la présence de structures embryonnaires qui n'ont pas leur homologue dans l'organisme adulte: il observe avec précision le développement embryonnaire de novo du cœur, des vaisseaux sanguins, ainsi que la formation du tube intestinal par plissement d'un tissu qui formait à l'origine l'un des «feuillets embryonnaires » : une notion que Pander et von Baer reprendront et développeront, et qui deviendra un concept fondamental de l'embryologie moderne. Cette dernière observation conduira Wolff à déclarer: «Si on apprécie correctement cette formation de l'intestin, je crois qu'il ne peut plus persister de doute du bien-fondé de l'épigenèse ».

Heinz Christian Pander travailla sur l'embryon de poulet pendant moins de deux ans et la rigueur de ses observations l'amena à formuler plusieurs notions clés de l'embryologie moderne. Il élabora en particulier la théorie des feuillets germinaux (ou feuillets embryonnaires), que Caspar Wolff avait déjà ébauchée, suivant laquelle les différentes parties de l'embryon se forment à partir de trois feuillets embryonnaires, que l'on connaît de nos jours sous les termes d'ectoderme, de mésoderme et d'endoderme. L'existence d'un stade du développement composé de trois feuillets de structure assez simple, intermédiaire entre l'œuf et un embryon plus tardif, au cours duquel les organes se mettent en place, allait bien sûr dans le sens d'une vision épigéniste et consacrait la fin prochaine de la théorie de la préformation dans sa forme initiale. D'autres observations de Pander étaient également en faveur de l'épigenèse, puisqu'il nota que les feuillets embryonnaires ne formaient pas leurs organes respectifs indépendamment les uns des autres. Au contraire, chaque feuillet germinatif «n'est pas encore suffisamment indépendant pour affirmer ce qu'il est véritablement; il a encore besoin de l'aide de ses compagnons de route, et c'est pourquoi, bien que destiné à d'autres fins, tous trois s'influencent mutuellement jusqu'à ce qu'ils aient atteint un niveau approprié » [6].
Karl Ernst von Baer, qui domina la science de son époque et est souvent considéré comme l'un des plus grands embryologistes du XIX $x^{e}$ siècle, poursuivit les travaux de son ami Pander sur l'embryon de poulet : l'importance de son œuvre finira par éclipser le travail de Pander. Von Baer décrit pour la première fois, la chorde, une structure axiale embryonnaire et transitoire d'origine mésodermique impliquée dans la mise en place du système nerveux. Dans son ouvrage Über Entwicklungsgeschichte der Tiere, publié en 1828, et qui marque le début d'une embryologie comparée sérieuse, von Baer décrit avec précision le développement embryonnaire des vertébrés, de la fécondation jusqu'à la naissance, et contribue à consolider la théorie de l'épigenèse, un demi-siècle après les travaux de Wolff. II reconnaît qu'il y a un schéma commun de développement partagé par tous les vertébrés, que l'organisation des feuillets est valable pour tous les embryons de vertébrés, et que les différents organes ont la même origine quelle que soit l'espèce (poisson, grenouille, poulet ou mammifère), puisqu'ils proviennent tous des feuillets embryonnaires.

Si ces brillantes études d'embryologie descriptives de la première partie du XIX ${ }^{\mathrm{e}}$ siècle mettaient sérieusement à mal la théorie de la préformation, les premières tentatives expérimentales de la fin du siècle allaient remettre au premier plan, dans une version plus élaborée, une vision préformationniste ${ }^{2}$ du développement $[2,7,8]$. Pour les embryologistes « expérimentalistes », il ne s'agissait plus, comme pour leurs prédécesseurs, de décrire le développement et la morphologie de l'organisme entier, mais de focaliser leurs analyses sur des évènements particuliers de l'ontogenèse. II s'agissait également de perturber expérimentalement ces événements, afin de mieux comprendre le fonctionnement normal de l'organisme en développement. En 1958, Louis Gallien (1908-1976) - le fondateur de la chaire d'embryologie de la Sorbonne en 1954 - notait avec justesse que «Deux grandes idées ont dominé l'ensemble des travaux de l'embryologie expérimentale. Elles correspondent aux théories Préformiste et Épigénétique du développement » [7]. En effet, car même si les expérimentalistes ont enterré la théorie de la préformation initiale on est maintenant bien loin de l'« homonculus » de Nicolas Hartsoeker ! - ; le débat entre tenants des deux visions, préformation et épigenèse, est encore loin d'être terminé. C'est le Français Laurent Chabry (1855-1894) qui fut un précurseur, mais également deux élèves de l'Allemand Ernst Haeckel (1834-1919), Wilhelm Roux (1850-1950) et Hans Driesch (1867-1941), qui contribuèrent à fonder l'embryologie expérimentale, appelée plus tard embryologie causale.

\footnotetext{
${ }^{2}$ Théorie biologique considérant que les êtres vivants sont formés complètement
} dans l'embryon. 


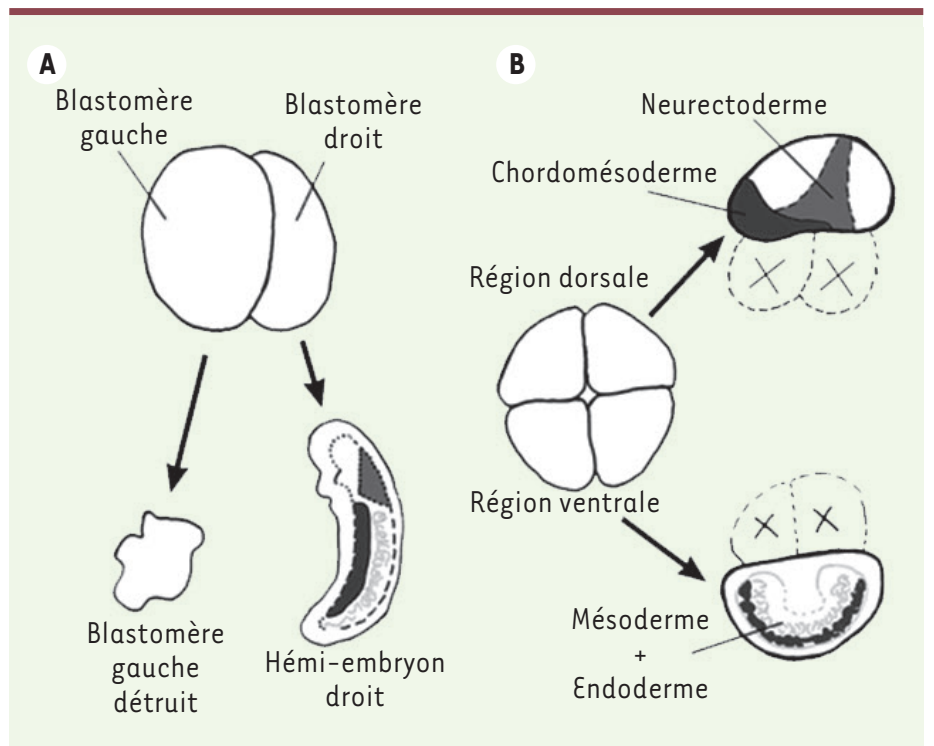

Figure 1. Expériences de Chabry. Destruction, au stade deux cellules, du blastomère gauche et formation d'un hémi-embryon droit (A). La destruction, au stade quatre cellules, des deux blastomères ventraux, conduit à la formation des structures dorsales comme le chordomésoderme et le neurectoderme; la destruction, au stade quatre cellules, des deux blastomères dorsaux conduit à l'apparition des formations msésodermiques et endodermiques, mais pas à celle de neurectoderme (B).

Laurent Chabry travaillait sur l'ascidie, un animal marin dont la pigmentation de l'œuf permet de reconnaître très tôt le plan de symétrie bilatérale du futur embryon. Il a étudié les embryons de l'ascidie Cynthia, dont on pouvait facilement se procurer des œufs et qui était alors un modèle de recherche très en vogue dans toute l'Europe. II est le premier, en 1887, à observer la formation anormale de demiembryons chez l'ascidie, anomalie résultant de l'absence de développement d'un des deux blastomères provenant de la première division de segmentation : en détruisant, avec une baguette de verre effilée, un des deux blastomères de l'embryon d'ascidie - situés de part et d'autre du plan de symétrie bilatérale - après la première division de segmentation, il reproduit expérimentalement la formation anormale de demi-embryons : le blastomère survivant donne un hémi-embryon ; le blastomère gauche donnant un hémi-embryon gauche, et le blastomère droit, un hémi-embryon droit (Figure 1A) [9].

Ce type d'expérience fut également réalisé à un stade plus avancé du développement. Au stade quatre cellules, par exemple, si les deux blastomères ventraux sont séparés des deux blastomères dorsaux, deux embryons anormaux et incomplets sont obtenus: I'un présente des structures uniquement ventrales et l'autre, des structures uniquement dorsales (Figure 1B). Ces expériences furent reprises et confirmées par la suite par l'embryologiste américain Edwin Grant Conklin (1863-1952) en 1905 [10]. Il concluait que la destinée de chaque blastomère est fixée dès le début de la segmentation. Ces faits expérimentaux montrent en effet qu'aucune régulation n'est possible à partir du stade deux blastomères et que l'on peut se représenter le très jeune embryon comme une mosaïque de territoires prédéterminés.
Chabry, s'opposant à tout retour à la théorie de la préformation, restait cependant un peu plus prudent dans ses conclusions de 1887.

Un an après ces expériences, en 1888, l'Allemand Wilhelm Roux (1850-1924), que l'on considère généralement comme le fondateur de la «mécanique du développement » - et dont l'objectif était la recherche expérimentale des causes présidant l'ontogenèse retrouve chez l'œuf fécondé de grenouille le même résultat que Chabry chez l'ascidie [11] : en détruisant un des deux blastomères issus de la première division de segmentation avec une aiguille portée préalablement à la flamme, il observe que la cellule touchée se dégrade alors que l'autre blastomère se divise pour donner, là aussi, un hémi-embryon (Figure 2). Ces observations conduisirent Roux à développer le concept d'œuf mosaïque. Les expériences de Chabry, de Roux puis de Conklin semblaient conforter une vision «préformationniste moderne » du développement embryonnaire et allaient dans le sens des théories développées par le biologiste allemand August Weismann (1834-1914).

August Weismann avait développé la théorie du plasma germinatif qui distingue d'une part les cellules germinales, chargées d'assurer la continuité de la descendance des individus au travers des gamètes et d'autre part, des cellules somatiques, responsables de la construction de l'organisme. Cette théorie fut vraiment à l'origine du concept de développement mosaïque, une version plus élaborée de la théorie préformationniste. $\varepsilon n$ effet, avec cette vision des choses, la différenciation des lignages somatiques est sous le contrôle de déterminants cytoplasmiques distribués de manière régionalisée dans l'œuf. Cela conditionne le devenir des cellules qui en héritent au fil des divisions cellulaires. Dans ce modèle de développement, la destinée d'une cellule est déterminée et conditionnée par son origine et l'embryon est constitué d'une mosaïque de territoires dont le devenir est déterminé.

Pour August Weismann, la différenciation des cellules somatiques est un phénomène irréversible qui l'a amené à postuler l'existence d'un lignage à part, la lignée germinale, responsable de la transmission héréditaire des caractères. Contrairement aux cellules somatiques, la différenciation des gamètes n'altère pas leur caractère totipotent, puisqu'elles restent capables de donner naissance à tous les territoires de l'embryon après la fécondation [12-13].

Pour Wilhelm Roux, comme le note Hendrik Cornelis Dirk de Wit (1909-1999) [2], les organes de l'animal adulte sont présents en instance, dès le début de la croissance, et leur fonction, leur structure et leur forme 


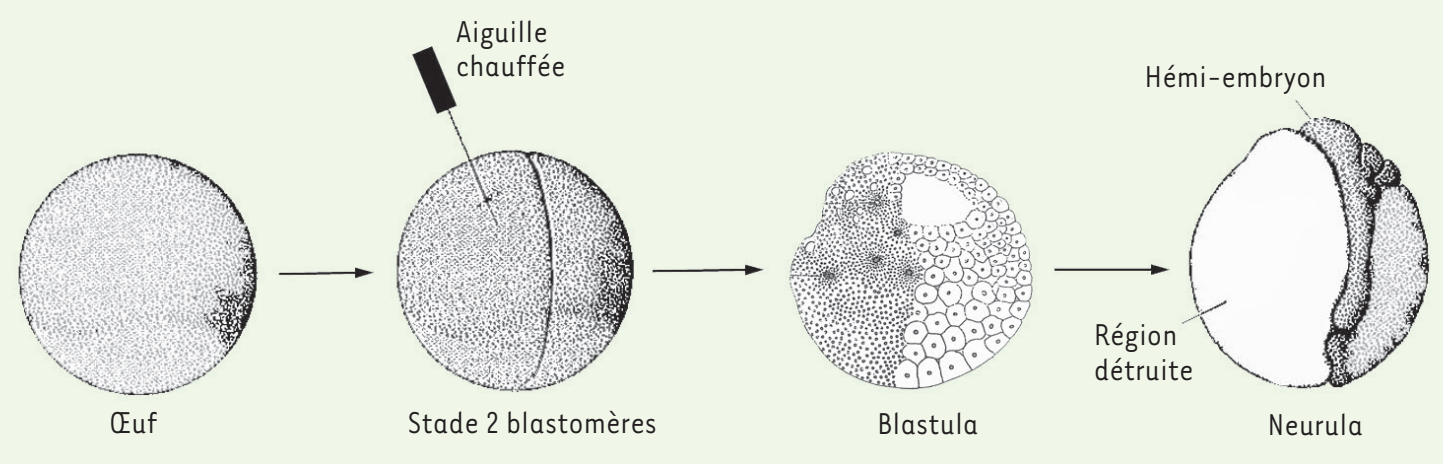

Figure 2. Expérience de Roux : destruction d'un blastomère chez la grenouille au stade deux blastomères, donnant un hémi-embryon.

sont alors déterminées, soumises à un développement autonome. La membrane cellulaire entre les deux premières cellules filles, nées de la première segmentation de l'œuf, détermine le plan de symétrie de l'adulte, ce qui implique que les deux cellules filles doivent posséder chacune la prédisposition complète de la totalité des organes pairs. En 1891, à la station marine de Trieste, aujourd'hui en Italie, Hans Driesch réalise une expérience chez l'embryon d'oursin qui invalide l'expérience de Roux. II utilise une technique mise au point peu de temps avant par les frères Oscar (1849-1922) et Richard (1850-1937) Hertwig, deux embryologistes allemands célèbres de l'époque. Elle consistait à séparer, par simple agitation mécanique, les blastomères d'un embryon au stade deux ou même quatre cellules, puis à les laisser se développer indépendamment. Driesch montre que chacun des blastomères se développe pour former un embryon normal, puis une larve d'oursin, Pluteus ${ }^{3}$, complète et harmonieuse, quoique de plus petite taille [14] (Figure 3).

Cette observation est en opposition avec ce que pouvait laisser prédire le résultat de l'expérience de Wilhelm Roux, à savoir le développement indépendant de deux hémi-embryons. L'expérience de Driesch montre que, chez la très jeune blastula d'oursin, chaque blastomère contient la totalité de l'information nécessaire pour un développement embryonnaire harmonieux, c'est-à-dire que toutes les cellules sont équipotentielles en termes de développement. À l'inverse de l'expérience de Roux, celle de Driesch montre que le jeune embryon est capable de régulation, ce qui invalide le concept préformationniste et est en faveur de la théorie épigénétique qui s'est imposée depuis. Driesch, déclare lui-même que la théorie de la préformation est une erreur et il souhaite remplacer la théorie de la préformation des esquisses initiales ( Theorie der Anlagenpräformation ») par une théorie de l'évolution épigénétique («Theorie der epigenetischen Evolution ») [15]. Driesch adresse à son maître, Ernst Haeckel, une « kritische Studie » (une étude critique) accompagnée d'une lettre dans laquelle il demandait si le développement embryonnaire ne pouvait pas être aussi envisagé sous cet éclairage : Haeckel ne se donna pas la peine de lui répondre directement mais il fit savoir par la bouche d'un

\footnotetext{
3 Le Pluteus est la première forme de l'oursin à la sortie de l'œuf.
}

ami commun que Driesch était mûr pour l'admission en asile psychiatrique [16]... Driesch subit également les foudres de Roux et, à une époque où l'on passait plus de temps en discussions qu'en expériences, le modèle de la mosaïque garda des défenseurs ardents. Ces derniers s'opposent alors au modèle de l'équipotentialité et le débat est vif entre partisans de chaque modèle. Driesch poursuit cependant, et avec détermination, ses expériences et montre que, sous l'effet d'une agitation modérée, les cellules de très jeunes embryons d'oursin se déplacent les unes par rapport aux autres, mais que la perturbation de la disposition cellulaire n'affecte en rien le développement ultérieur, confirmant l'équipotentialité de ces cellules [17].

Il faut cependant noter que même s'il énonçait en 1894, à partir de ses travaux sur l'oursin, que la formation de l'embryon était le résultat d'une succession bien ordonnée de réactions chimiques à l'intérieur des cellules et que la croissance embryonnaire était régie par une « induction » chimique [15], Driesch ne valida jamais la possibilité d'une interprétation mécanistique de l'ontogenèse et formula en 1908, dans sa Philosophie de l'Organisme [18], une théorie vitaliste, dans laquelle un facteur transcendant, l'«Entéléchie » ${ }^{4}$, joue un rôle capital. Pour Driesch, pour qui les phénomènes biologiques ne peuvent pas être réductibles aux seules lois physico-chimiques, ce facteur transcendant permettrait d'expliquer les phénomènes vitaux et contrôlerait le développement embryonnaire. De ce point de vue, sa pensée allait dans le sens de la conception vitaliste développée en son temps par Aristote. II heurte cependant la majorité des zoologistes, anatomistes et embryologistes de l'époque, presque tous adeptes d'un naturalisme scientifique positif. Beaucoup d'entre eux font remarquer que la régulation n'est pas un fait

\footnotetext{
${ }^{4}$ Principe créateur de l'être.
} 


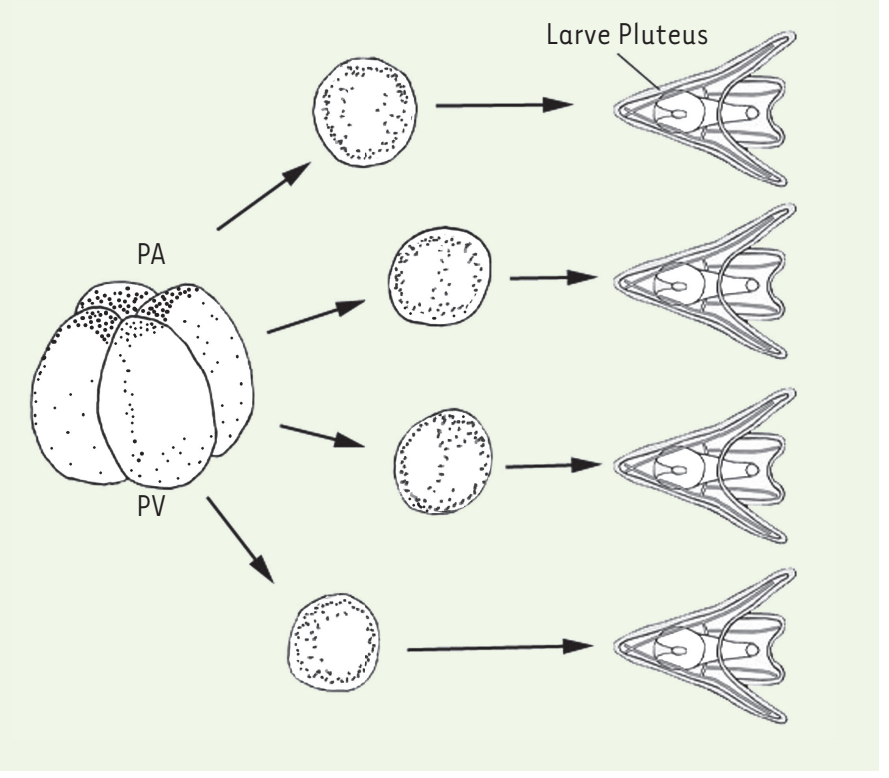

Figure 3. Expérience réalisée par Driesch chez l'oursin au stade quatre blastomères. Chacun des blastomères est à l'origine d'une larve Pluteus d'oursin complète et harmonieuse. PA : pôle animal ; PV : pôle végétatif.

général : les tuniciers (comme l'ascidie), comme le montraient Chabry et Conklin, les gastéropodes et les vers, ne semblent pas la montrer et elle ne pourrait être causée que par l'expérience, dans les travaux de Driesch : il n'est pas sûr que les phénomènes provoqués expérimentalement interviennent dans le développement normal. La plupart des chercheurs de l'époque réclament donc, avant de suivre Driesch dans ses conclusions hardies, des analyses plus approfondies. Driesch, jeune chercheur de 24 ans, persistera dans ses conclusions, mais il finira par démissionner et quittera le domaine de l'embryologie pour se consacrer à la philosophie.

Progressivement cependant, l'expérimentation donnera raison à Driesch et, dans les années qui suivirent, l'équipotentialité cellulaire dans les phases précoces du développement fut confirmée par plusieurs chercheurs, dans différents modèles animaux. Oscar Hertwig, qui obtenait parfois, en 1893 , des embryons entiers en répétant l'expérience de Wilhelm Roux, rejeta le modèle de la mosaïque [19]. En revanche, Hertwig soutenait que le contenu de l'œuf devait être interprété comme équipotentiellement isotopique, c'est-à-dire comme une masse homogène, ce qui allait s'avérer inexact, comme le montreront les expérimentations ultérieures. L'embryologiste américain Edmund B. Wilson (1856-1939), en particulier, se livra à une vive critique de la théorie de l'œuf mosaïque de Roux, en utilisant un petit animal marin chordés, l'Amphioxus, comme modèle d'étude [20]. On sait maintenant que les résultats de l'expérience de Roux étaient dus à un artefact expérimental lié à la présence persistante de la matrice extracellulaire de la cellule détruite. Chez le triton, Amedeo Herlitzka (1872-1949) [21] et Hans Spemann (1869-1941) [22-24] montrèrent que si on sépare par ligature les deux premiers blastomères, on peut obtenir à partir de chacun d'entre eux un embryon complet (dans la mesure cependant où le premier plan de segmentation coïncide avec le plan de symétrie bilatérale). En 1895, Thomas Hunt Morgan (1866-1945), qui s'intéressa à la mécanique du développement et à l'embryologie expérimentale, avant ses études sur la transmission des caractères héréditaires (qui lui valurent le Prix Nobel de physiologie ou médecine en 1933), montre également, chez la grenouille, que les blastomères sont doués d'un «pouvoir régulateur », comme ceux de l'oursin [25]. Chez l'ascidie, II faudra attendre les travaux du Belge Albert Dalcq (1893-1973), en 1932, pour montrer que si l'on fractionne un œuf vierge en deux parties, en effectuant une ligature méridienne, chacune des moitiés de l'œuf peut être fécondée par un spermatozoïde et donner par la suite un embryon complet [26]. Avant la fécondation, l'œuf d'ascidie n'est donc pas encore une mosaïque, et peut manifester une capacité de régulation. En fonction de l'espèce, il apparaît que le programme de développement est fixé plus ou moins tôt, permettant des phénomènes de régulation plus ou moins précoces.

L'ensemble des expériences initiées par Hans Driesch aura donc permis, d'une part, d'en finir définitivement avec toute forme de concept préformationniste et, d'autre part, d'ouvrir et de développer de nouveaux champs d'investigation concernant les rapports entre noyau et cytoplasme, l'hétérogénéité du cytoplasme, et le concept de gradient. $\diamond$

\section{SUMMARY}

Hans Driesch (1867-1941) and the end of any preformationist vision of development

While the work of early 19th century embryologists marked the end of preformation theory in its initial form, the first experimental attempts at the turn of the century led to the development of the concept of the mosaic egg - a more elaborate preformationist view of development - in which the embryo is made up of a mosaic of territories with a determined future and subjected to autonomous development. By separating sea urchin blastomeres at 2/4 cell stages, a young researcher, Hans Driesch shows that each of the blastomeres develops to form a complete and harmonious larva and that the young embryo is therefore capable of regulation. Thus this invalidated the concept of mosaic development, which predicted the independent development of two hemi-embryos and it opened up new fields of investigation for modern embryology. $\diamond$

\section{LIENS D'INTÉRÊT}

L'auteur déclare n'avoir aucun lien d'intérêt concernant les données publiées dans cet article. 


\section{RÉFÉRENCES}

1. Louis P. Aristote. De la génération des animaux : texte établi et traduit. Paris : Les Belles Lettres, $1961: 440$ p.

2. de Wit HCD. Histoire du développement de la biologie, volume II. Lausanne : Presses polytechniques et universitaires romandes, $1993: 460 \mathrm{p}$.

3. Chanoine C. Histoire de la biologie du développement. Paris : Ellipses, $2018: 192$ p.

4. Hartsoeker N. Essay de dioptrique. Paris : J. Anisson Ed, 1694 : 180 p.

5. Gautier d’Agoty J. Zôos-génésie, ou Génération de l'homme et des animaux. Paris : J. Bullot Ed, $1750: 20$ p.

6. Pander HC. Beiträge zur Entwickelungsgeschichte des Hühnchens im Eye. Wurtzbourg : $1817: 42$ p.

7. Gallien L. Problèmes et concepts de l'embryologie expérimentale. Paris : Gallimard, $1958: 586 \mathrm{p}$.

8. Pourquié 0. Biologie du développement. Paris : Hermann, $2002: 220$.

9. Chabry L. Contribution à l'embryogénie normale et tératologique des Ascidies simples. J Anat physiol 1887 ; 23 : 167-319.

10. Conklin EG. Mosaic development in ascidian eggs. J Exp Zool $1905 ; 2$ : 145-223.

11. Roux W. Über die künstliche Hervorbringung halber Embryonen durch Zerstörung einer der beiden ersten Furchungskugeln, sowie über die Nachentwicklung (Postgeneration) der fehlenden Körperhälfte. Virchows Arch $1888 ; 114: 133-53$.

12. Weismann A. Die Continuitât des Keimplasma's als grundlage einer Theorie der Vererbung. léna G. Fisher Ed, $1885: 122 \mathrm{p}$.

13. Weismann A. Das keimplasma Eine Theorie der Veerbung. léna : G. Fisher Ed, $1892: 622$ p.

14. Driesch H. Entwicklungsmechanistische Studien I. Der Werth der beiden ersten Furchungzellen in der Echinodermenentwicklung. Experimentelle Erzeugung von Theilund Doppelbildungen. Zeit Wiss Zool 1891; 53 : 160-84.

15. Driesch H. Analytische theorie der organischen entwicklung. Leipzig : W. Engelmann Ed, 1894 $184 \mathrm{p}$.

16. Oppenheimer JM. Essays in the history of embryology and biology. Cambridge : MIT Press, 1967 : $374 \mathrm{p}$.
17. Driesch H. Zur Verlagerung der Blastomeren des Echinideneies. Anat Anz $1893 ; 8: 348-57$.

18. Driesch $H$. The Science and Philosophy of the Organism. London : A and C Black Ed, 1908 : 322 p.

19. Hertwig 0 . Uber den Werth der ersten Furchungszellen fur die Organbildung des Embryos. Experimentelle Studien am Frosch und Tritonei. Arch Mikrosc Anat $1893 ; 42: 662-807$

20. Wilson $\varepsilon B$. Amphioxus, and the mosaic theory of development. J Morph $1893 ; 8: 579-638$

21. Herlitzka A. Contibuto allo studio della capacita evolutiva dei due primi blastomeri nell uovo di tritone (Triton cristatus). Roux'Arch Entw 1896 ; 2 : 352-69.

22. Spemann H. Entwicklungsphysiologische Studien am tritonei. I. Roux'Arch Entw $1901 ; 12$ : 224-64.

23. Spemann H. Entwicklungsphysiologische Studien am triton-Ei. II. Roux'Arch Entw 1902 ; $15: 448-534$.

24. Spemann H. Entwicklungsphysiologische Studien am triton- $\varepsilon$ i. III. Roux'Arch Entw 1903; 16:551-631.

25. Morgan TH. Half-embryos and whole-embryos from one of the first two blastomeres of the frog's egg. Anat Anz $1895 ; 10: 623-8$.

26. Dalcq A. Etude des localisations nucléaires dans l'œuf vierge d'Ascidie par des expériences de mérogonie. Arch Anat Micr Morph Exp $1932 ; 28$ : 224-9.

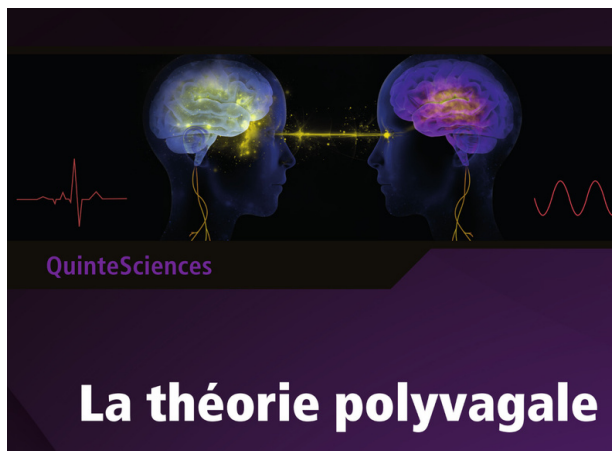

Fondements neurophysiologiques des émotions, de l'attachement, de la communication et de l'autorégulation

Stephen W. Porges

Traduit par Nico Milantoni et Isabelle Chosson-Argentier

\section{edpsciences}

\section{Enfin disponible en français}

\section{L'ouvrage de référence de S.W.Porges}

TIRÉS À PART

C. Chanoine
S.W.Porges est le spécialiste mondial du lien unissant le système nerveux autonome au comportement social. Il nous offre de passionnantes perspectives sur la façon dont notre système nerveux autonome gère inconsciemment notre engagement social, la confiance, l'intimité.

La traduction a été réalisée par des experts : Nico Milantoni est psychologue, praticien et formateur de la méthode Hipérion. Isabelle Chosson-Argentier est Docteur en pharmacie, conseil en nutrition, micronutritionniste, phyto-et aromathérapeute, praticienne de la méthode Hipérion.

ISBN : 978-2-7598-2498-4 373 pages - $69 €$ TTC

En vente sur la boutique.edpsciences.org 